\title{
Isotropic pressure promoted collective self-healing response in granular molecular crystals
}

\author{
Yuancun Qiao ${ }^{1,2 \dagger}$, Changzeng Fan $^{3 \dagger}$, Yuwei $\mathrm{Li}^{2}$, Kai Wang ${ }^{1 *}$ and Bo Zou ${ }^{1 *}$
}

The ability to spontaneously heal injuries is an important survival feature for humans, plants, and animals in nature. Inspired by nature, man-made self-healing materials that are capable of repairing damage and degraded properties either autonomously or triggered by external stimuli have been developed to improve the lifetime, safety, and functionality of products [1-3]. To date, most self-healing materials are representative soft materials, such as polymers and gels [4-6]. Relative to highly elastic and adaptive soft materials, long-range ordered molecular crystals are rigid and unadaptable and are highly vulnerable to stress-induced cracks. Accordingly, the selfhealing property is meaningful for ordered molecular crystals as it can endow molecular crystals with the ability to resist the loss of functionality and service lifetime during specific applications.

However, ordered molecular materials with self-healing property are rarely studied. In 2016, Naumov et al. [7] reported the first self-healing behavior in dipyrazolethiuram disulfide molecular crystals and extended the self-healing concept to ordered molecular crystals. Thus far, only a few self-healing molecular crystals have been reported [7-12]. Additionally, molecular materials show limited self-healing efficiency. For instance, the selfhealing degree of dipyrazolethiuram disulfide is only $7 \%$ [7], and that of boronic ester is only $67 \%$ [11]. Hence, the fabrication of self-healing molecular crystals with high efficiencies is of great significance but is still challenging.

In this work, we report the first case of isotropic pressure-triggered and -promoted self-healing behaviors in granular $N$-methylurea (NMU) crystals with a high efficiency. Under ambient condition, NMU crystallizes in orthorhombic, space group $P 2_{1} 2_{1} 2_{1}$, with four molecules per unit cell [13]. As shown in Fig. 1a, the NMU molecule comprises an inactive methyl group and abundant hydrogen bonding donors and acceptors that show a typical rigid-soft model. When viewed along the $a$ axis, the NMU crystal is formed by a series of interconnected rectangular-like motifs (Fig. 1b). For an individual rectangular motif, the sides and corners are constituted by $\mathrm{N}-\mathrm{H}$... $\mathrm{O}$ hydrogen bonds and NMU molecular skeletons, respectively (Fig. 1c, d). Moreover, the NMU molecular skeletons at the corners are connected by bifurcated hydrogen bridges, forming an infinite chain along the $a$ axis (Fig. 1e, f). Methyl groups are located at the center of the rectangular motif, and they are surrounded by numerous voids for the inert bonding capability of the methyl groups.

The whole process of self-healing is directly monitored by optical microscopy experiments (Fig. 2). At the beginning, the initial chamber is not fully transparent because of the reflecting and scattering effects occurring between a mass of randomly existing grain boundaries (GBs) in NMU powder crystals. With increasing pressure, the chamber slightly becomes translucent, thereby indicating that the NMU grains gradually come into contact with one another under pressure, as often observed in high-pressure experiments. When the pressure reaches $0.24 \mathrm{GPa}$, surprisingly, the NMU grains at the chamber edge immediately fuse, showing an impressive self-healing behavior. Remarkably, a distinct phase boundary between the parental sample and the daughter product shows a domino-like progression during the self-healing process. At last, the dynamic fusion homogeneously diffuses throughout the entire sample chamber with a macroscopic transformation from powder crystals to an integrated crystal and a drastic pressure-dropping behavior (Video S1). The chamber becomes significantly

\footnotetext{
${ }^{1}$ State Key Laboratory of Superhard Materials, College of Physics, Jilin University, Changchun 130012, China

${ }^{2}$ North China Institute of Aerospace Engineering, Langfang 065000, China

${ }^{3}$ State Key Laboratory of Metastable Materials Science and Technology, Yanshan University, Qinhuangdao 066004, China

${ }^{\dagger}$ These authors contributed equally to this work.

* Corresponding authors (emails: kaiwang@jlu.edu.cn (Wang K); zoubo@jlu.edu.cn (Zou B))
} 


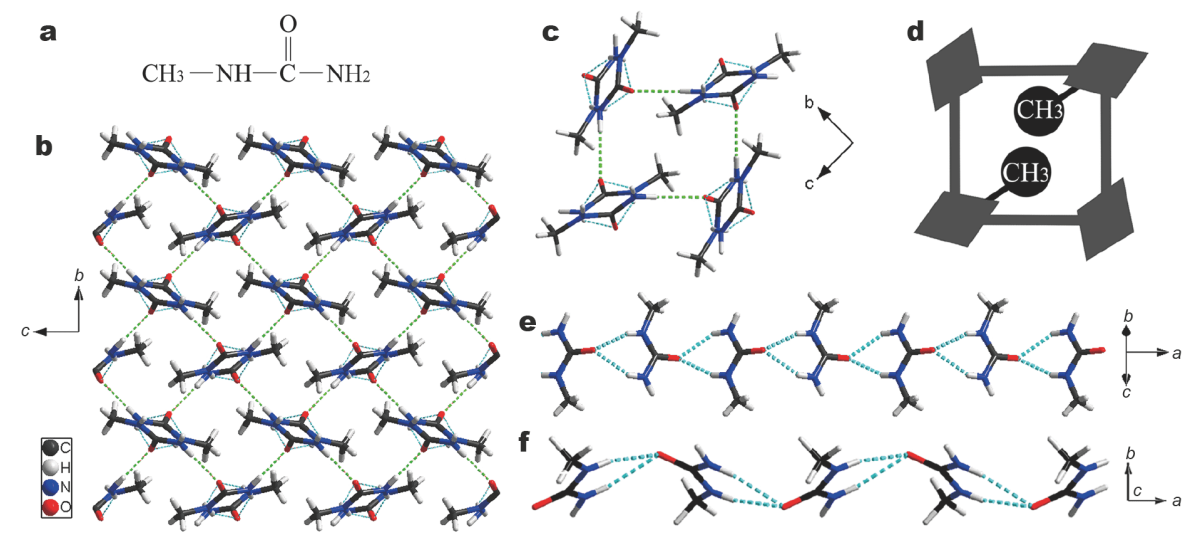

Figure 1 Molecular and crystal structures of NMU. (a) Molecular structure of NMU. (b) Crystal structure and hydrogen bonding networks of NMU crystal at ambient pressure viewed along the $a$ axis, showing rectangular motifs. (c) An individual rectangular motif. (d) The diagrammatic drawing of rectangular motif. (e) The bifurcated hydrogen bridges that form an infinite chain along the $a$ axis. (f) The zigzag infinite chain viewed along the $c$ axis.

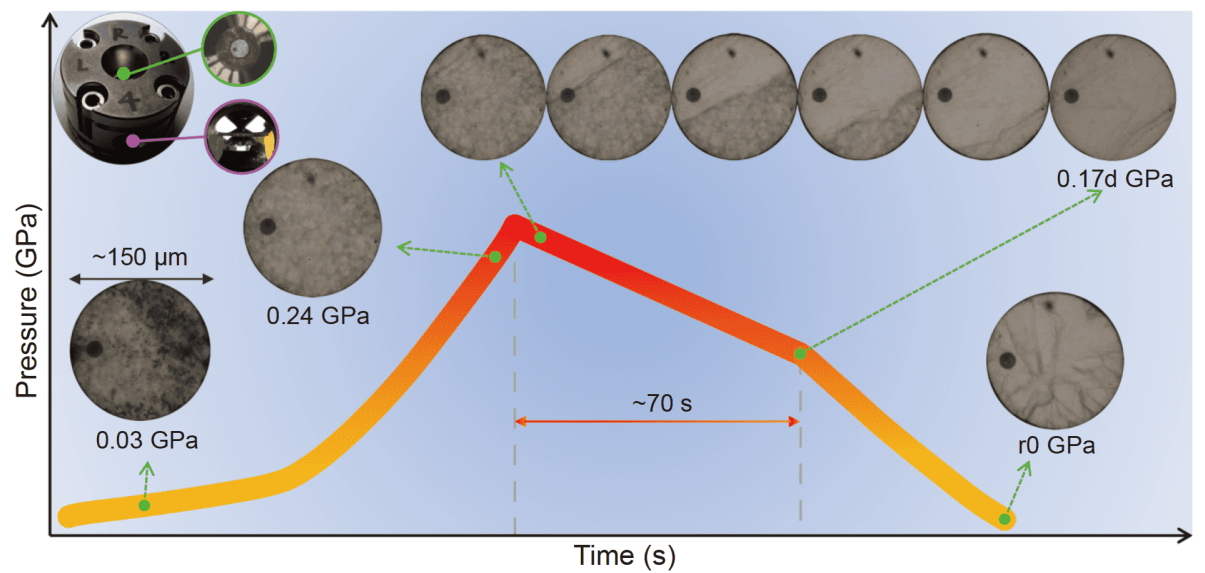

Figure 2 In situ optical microscopy of NMU powder crystals under pressure, showing a typical self-healing process. Mechanical pressure is generated through a diamond anvil cell (DAC) apparatus which is shown in the upper left. The " $\mathrm{d}$ " in " $0.17 \mathrm{~d}$ " represents that the pressure drops to $0.17 \mathrm{GPa}$ from a higher pressure point after the self-healing process. Typical recording of self-healing process can be viewed in Video S1.

bright after the self-healing process. This result suggests the disappearance of abundant random GBs as a result of grain fusion. As shown in Fig. S1, the high-pressure transmission experiment indicates that the optical transmissivity exhibits an abrupt change from $\sim 14 \%$ to $~ 85 \%$ during the self-healing process, which confirms the disappearance of GBs and the occurrence of grain fusion behavior during the self-healing process. After releasing pressure, the terminal single crystal maintains integrity, except for some subboundaries within it, suggesting the existence of another high-efficiency self-healing effect during the pressure-releasing process. Although the selfhealing products in pressure-elevating and pressure-releasing processes are nearly perfect single crystals, they break under further pressure treatment, which is evi- denced by the appearance of parallel cracks (Fig. S2f, g). The self-healing behavior in NMU is only triggered by isotropic pressure at room temperature, which is different from the self-healing effect in previously reported molecular crystals that is triggered by high temperature or uniaxial stress. The other characteristics of the self-healing property of NMU are presented in Fig. S2, Table S1, and Videos S2-5.

To further confirm the self-healing and understand the origin of GB fusion and migration motions in NMU, we used in situ single-crystal X-ray diffraction (SXRD) experiments to characterize the structural evolution during the self-healing process. As shown in Fig. 3a, the initial two-dimension (2D) XRD pattern is a typical circular pattern for powder X-ray diffraction (PXRD), which in- 

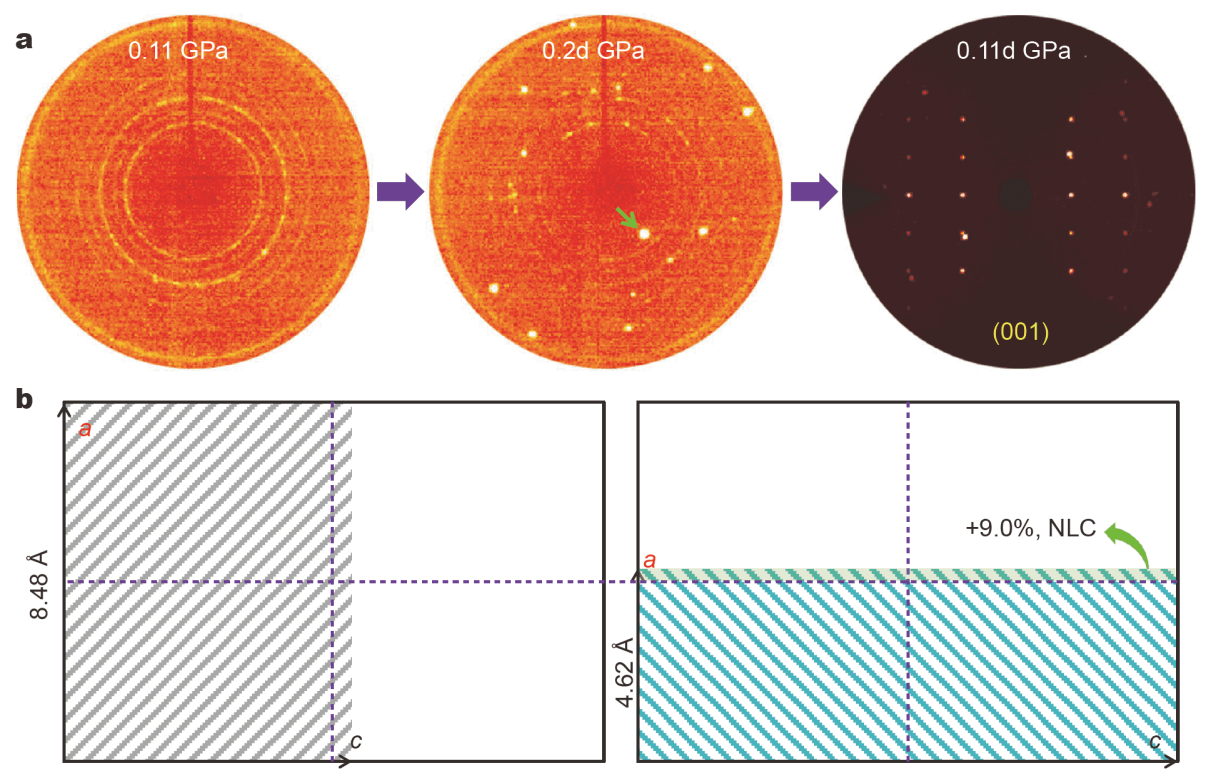

$6.92 \AA$

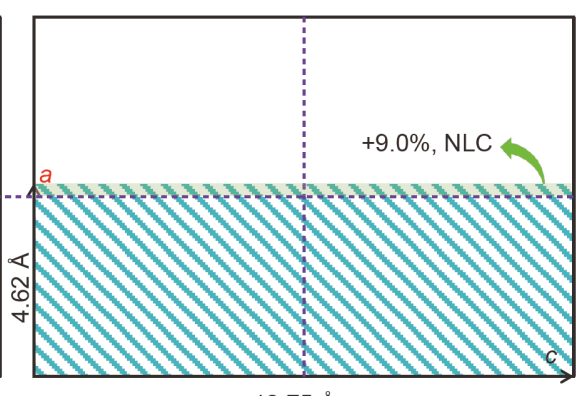

$12.75 \AA$

Figure 3 In situ SXRD experimental data of NMU powder crystals under pressure. (a) The variation of 2D SXRD images during the self-healing process. (b) The lattice information before (left, phase I) and after (right, phase II) self-healing process showing an anisotropic phase transition as a result of an NLC effect along the $a$ axis.

dicates that the initial sample is powder crystals corresponding to the two hours of grinding time from single crystals. With increasing pressure, some bright diffraction dots appear with co-existence of the circular pattern and the dotted pattern for SXRD, thereby signifying the occurrence of the self-healing process. When the self-healing process is completed, the circular pattern totally changes to a bright dotted pattern, which suggests the ordering of randomly distributed small grains and the formation of a large-sized single crystal. This result is in agreement with the optical microscopy experiments. The high quality of the SXRD pattern combined with the in situ optical images confirms the pressure-triggered selfhealing behavior in NMU. It also indicates that the selfhealing degree in NMU can be determined to be nearly $100 \%$. As shown in Fig. 3a, a new d value labeled with green arrow that is larger than all the $d$ values of phase I appears when the self-healing process occurs, which indicates that the self-healing process is accompanied by a phase transition. To investigate the structural difference before and after the phase transition, we obtain the crystallographic details of the self-healing product on the basis of in situ high-pressure SXRD data. Further analysis indicates no changes in the crystal system and space group, but large changes in cell dimensions are observed. To facilitate the comparison, the cell parameters of the two phases before (phase I) and after (phase II) the phase transition or self-healing process are described in Table S2. During the self-healing process, the total volume contracts $4.7 \%$. The dimensions along the $b$ and $c$ axes contract $5.4 \%$ and $7.8 \%$, respectively, while that along the $a$ axis expands $9.0 \%$, showing a negative linear compressibility (NLC) effect (Fig. 3b) [14,15]. Therefore, the phase transition is dramatically anisotropic. The selfhealing behavior and anisotropic phase transition occur simultaneously, which suggest that the anisotropic phase transition is a prerequisite for the self-healing process in NMU.

To gain further insights into the origin of the selfhealing behavior of NMU, in situ PXRD experiment with pressure-transmitting medium (PTM) was also carried out and the crystal motions were monitored simultaneously. According to the in situ optical microscopy images (Fig. 4a), the morphology of the sample in the chamber remains the same up to $2.05 \mathrm{GPa}$, and the selfhealing phenomenon is observed neither in small-sized uniform grains (grinding of $2 \mathrm{~h}$ ) nor in large-sized inhomogeneous grains (grinding of $5 \mathrm{~min}$ ) (Fig. S3), while there still exists a phase transition at about $0.19 \mathrm{GPa}$ which is evidenced by the discontinuity of the in situ PXRD patterns (Fig. 4b). The high-quality circular 2D PXRD patterns before and after the phase transition indicate that the self-healing process is not triggered (Fig. 4c-e), which is accordance with the in situ optical 

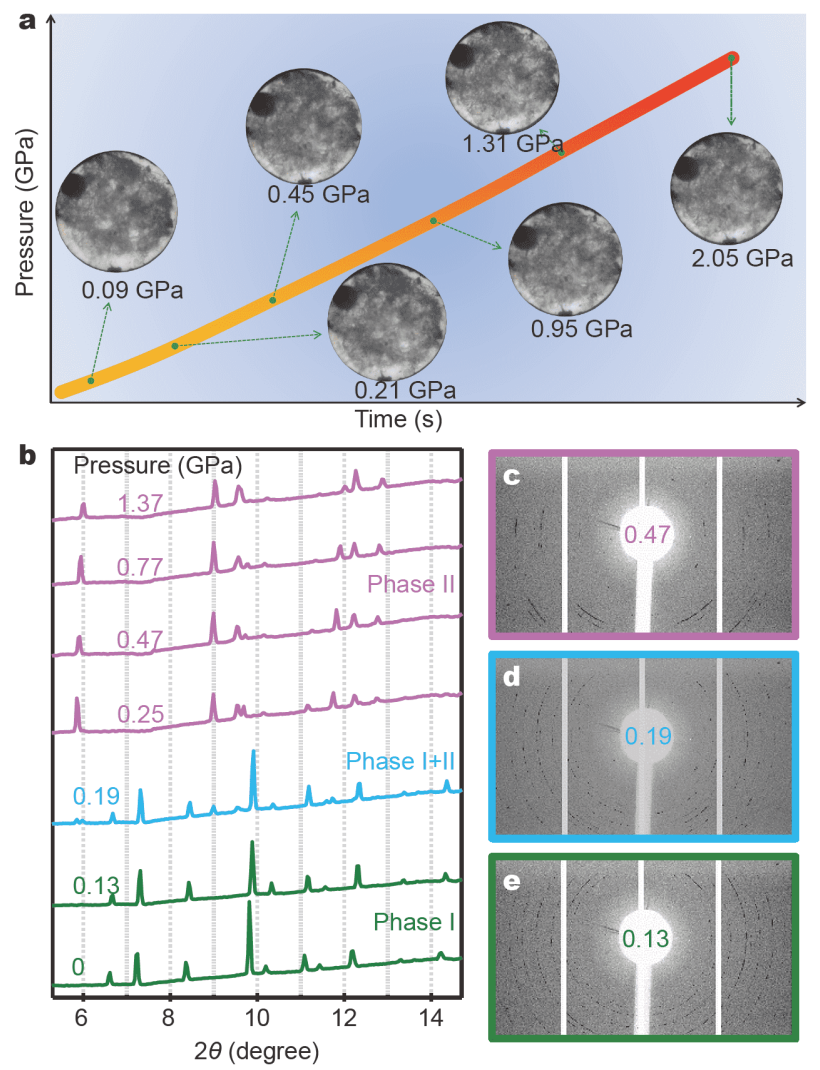

Figure 4 In situ PXRD experimental data of NMU powder crystals under pressure with PTM. (a) In situ optical microscopy of NMU powder crystals with PTM under pressure, showing the absence of selfhealing response. (b) In situ PXRD patterns of NMU crystal with PTM under high pressure. (c-e) 2D PXRD images at three selected pressure points.

microscopy images. Although the phase transition pressure with PTM is higher than that in the self-healing process, the phase transformation paths in two different experiments are the same. This is proved by high-pressure Raman experiments (Fig. S4), which intuitively indicates that the close contact of grains is another prerequisite for pressure-promoted self-healing in NMU. During the self-healing process, the initial NMU powder crystals must transform into a polycrystalline-like state without obstruction from PTM. Furthermore, the disappearance of dramatic pressure dropping indicates that external pressure is not only a trigger but also a fuel for the self-healing process.

Apart from NMU undergoing large morphological change caused by anisotropic phase transitions, the substantial difference between two phases (anisotropic phase transition) or a lattice mismatch usually results in tremendous changes in the crystal interior and morphology of mechanically responsive materials, such as thermo- salient (TS) and photosalient (PS) single crystals [16]. In TS and PS effects, the anisotropic phase transition or lattice mismatch is also a prerequisite and accounts for the generation of shear stress that drives various mechanical motions [17-21]. To confirm the shear stress generated from the anisotropic phase transition in NMU, we further performed low-temperature optical microscopy experiments not glued to DAC and the mechanical motions of NMU single crystal were monitored. The optical microscopy experimental data indicate that the NMU single crystal does undergo various TS effects, such as crystal jumping, moving and separation (Video S6) at about $110 \mathrm{~K}$, which is in accordance with the negative thermal expansion (NTE) effect under cooling (Fig. S5) [22]. Therefore, the anisotropic phase transition or anisotropic lattice evolution is capable of generating shear stress within the NMU crystal and further triggering the TS effects. For NMU under pressure, the GB migration, a typical mechanical motion, is also propelled by a sharp pressure gradient resulting from the shear stress generated by the anisotropic phase transition. We thus believe that the pressure-promoted self-healing process in NMU powder crystals owns a microcosmic mechanism similar to the TS and PS effects, except for the action style of stimuli and action time. The stimuli in TS and PS effects are a contactless action. While the stimulus, pressure, is a contact action in pressure-promoted self-healing. The TS and PS effects are instantaneous within a single crystal. However, pressure-promoted self-healing is a collective effect of a mass of small grains.

Drawing on the similarities and differences with PS and TS effects, we propose an synergistically external-pressure- and interior-stress-driven mechanism suitable for the self-healing process in NMU granular crystals (Fig. 5). At first, an individual NMU grain firstly undergoes an anisotropic phase transition triggered by external pressure, resulting in the accumulation of interior stress within the grain and concomitant sharp pressure gradient within the chamber (Fig. 5a). However, the separated NMU grain cannot exhibit displacive mobility for the isotropic space limitations generated by the DAC technique. In order to release the interior shear stress, it propels the phase boundary seated between two phases to migrate along a habit direction until a complete singlecrystal-to-single-crystal (SCSC) transformation (Fig. 5a). However, residual interior shear stress and pressure gradient still exist because the ability of releasing interior shear stress during phase boundary migration is far less powerful than macroscopic mechanical motions. Accordingly, the interior shear stress and pressure gradient 


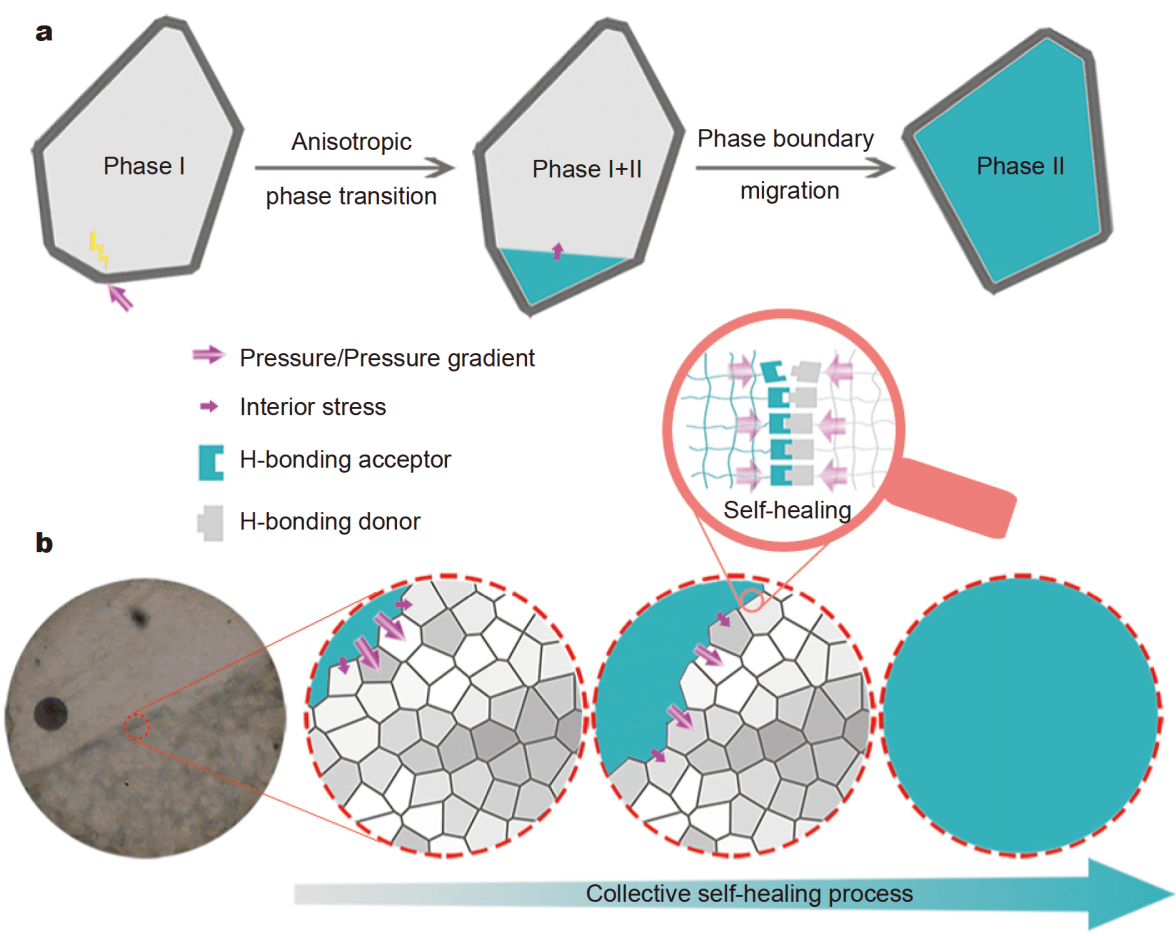

Figure 5 Schematic mechanism representation of the self-healing process in NMU granular crystals. (a) Anisotropic phase transition in an individual grain. (b) Collective self-healing process through re-formation of hydrogen bonds and GB migration process in selected region.

propel the phase boundary to adjacent NMU grains. When the interior shear stress reaches the GB position, the $\mathrm{C}-\mathrm{O}$ repulsion and $\mathrm{N}-\mathrm{H}: \mathrm{O}$ segment from two molecules on both sides of the GBs under the disturbance of interior shear stress and the compression effect from external pressure are coupled with each other through the formation of $\mathrm{N}-\mathrm{H} \cdots \mathrm{O}$ hydrogen bonds, finishing the selfhealing process. Therefore, the coupling of intermolecular and intramolecular interactions is the microscopic driving force for the collective self-healing behavior in NMU, which shows a similar coupling behavior between intermolecular and intramolecular interactions in water during regelation and instant ice formation [23,24]. The interior shear stress is thus fully released, and the rest of the chemical energy required for bonding is further provided by the external pressure. At last, domino-like GB fusion as a result of the collective self-healing effect of all grains further proceed through the continuous consumption of external pressure via an impressive transformation from mechanical energy to chemical energy, which is the reason of pressure-dropping and high selfhealing efficiency (Fig. 5b).

A typical way to construct a self-healing material is introduction of hydrogen bonds due to the reconstruction property of hydrogen bonds [25-28]. In NMU, the self- healing behavior also stems from the generation of hydrogen bonds between two separated grains as a result of anisotropic phase transition. In order to figure out the origin of the anisotropic phase transition, the molecular packing changes before and after phase transition should be presented. However, the data from the SXRD experiments are not enough to support the structural analysis because of the limitation from DAC apparatus. Thus, high-pressure Raman experiments were conducted to qualitatively depict molecular packing changes at the molecular level. Further Raman data indicate that the abundant hydrogen bonding sites in NMU molecules and steric-hindrance effect of methyl group are cooperatively responsible for the unique anisotropic phase transition in NMU crystals under external pressure. The rearrangement of hydrogen bonding networks brings the flattening of the zigzag infinite bifurcated $\mathrm{N}-\mathrm{H} \cdots \mathrm{O}$ chain along the $a$ axis, which further results in the NLC effect along the $a$ axis (Fig. S6). Further analysis of the Raman variation is presented in Fig. S4. Consequently, the key to the selfhealing of granular NMU is the mismatched lattice during the anisotropic phase transition. The anisotropic phase transition brings a mismatched lattice with a misfit stress, which propels the migration of GBs and results in the self-healing behavior. Compared with the original 
sample, the final product shows an enhanced light transmissivity for the growth of grain size. Therefore, the self-healing effect of granular NMU in this work can be used as a model to understand the dynamic nature of GB fusion and provides a theoretical guidance for the fabrication of functional films (such as perovskite solar cell films) with high quality and long-range uniformity. Additionally, the transformation from granular crystals to an integrated single crystal also suggests possible applications at pressure-driven actuators of light-based devices and the specific mechanosynthesis route for single-crystal growth through simple and green pressure treatment [29].

In summary, we elaborate an isotropic pressure-triggered and -promoted collective self-healing response in granular NMU crystals. The transformation from granular crystals to an integrated single crystal and the total change from a circular XRD pattern to a bright dotted XRD pattern synergistically confirm the self-healing response and also indicate the high-efficiency property, which provides an idea for improving the self-healing efficiency of other self-healing molecular crystals. External pressure is consumed and used to provide the chemical energy for the re-formation of $\mathrm{N}-\mathrm{H} \cdots \mathrm{O}$ hydrogen bonds during the self-healing process, which is the reason of pressure dropping and high efficiency. The self-healing effect with the transformation from powder crystals to single crystal in NMU depicts potential applications in the fields of pressure-driven actuators, specific single-crystal growth and optoelectronic film fabrication.

Received 15 January 2021; accepted 1 March 2021; published online 17 May 2021

1 White SR, Sottos NR, Geubelle PH, et al. Autonomic healing of polymer composites. Nature, 2001, 409: 794-797

2 Zwaag Svd. Self Healing Materials: An Alternative Approach to 20 Centuries of Materials Science. Delft: Springer, 2007

3 Mobaraki M, Ghaffari M. 2-basics of self-healing composite materials. In: Self-healing Composite Materials. 2020, 15-31

4 Yang Y, Urban MW. Self-healing polymeric materials. Chem Soc Rev, 2013, 42: 7446-7467

5 Urban MW, Davydovich D, Yang Y, et al. Key-and-lock commodity self-healing copolymers. Science, 2018, 362: 220-225

6 Wei Z, Yang JH, Zhou J, et al. Self-healing gels based on constitutional dynamic chemistry and their potential applications. Chem Soc Rev, 2014, 43: 8114-8131

7 Commins P, Hara H, Naumov P. Self-healing molecular crystals. Angew Chem Int Ed, 2016, 55: 13028-13032

8 Karothu DP, Weston J, Desta IT, et al. Shape-memory and selfhealing effects in mechanosalient molecular crystals. J Am Chem Soc, 2016, 138: 13298-13306

9 Liu G, Liu J, Ye X, et al. Self-healing behavior in a thermo- mechanically responsive cocrystal during a reversible phase transition. Angew Chem Int Ed, 2017, 56: 198-202

10 Gupta P, Karothu DP, Ahmed E, et al. Thermally twistable, photobendable, elastically deformable, and self-healable soft crystals. Angew Chem Int Ed, 2018, 57: 8498-8502

11 Commins P, Al-Handawi MB, Karothu DP, et al. Efficiently selfhealing boronic ester crystals. Chem Sci, 2020, 11: 2606-2613

12 Naumov P, Chizhik S, Commins P, Boldyreva E. Bending, jumping, and self-healing crystals. In: Mechanically Responsive Materials for Soft Robotics. Weinheim: Wiley-VCH Verlag GmbH \& Co. KGaA, 2020, 105-138

13 Huiszoon C, Tiemessen GWM. Monomethylurea: A redetermination. Acta Crystlogr B Struct Sci, 1976, 32: 1604-1606

14 Baughman RH, Stafstrom S, Cui C, et al. Materials with negative compressibilities in one or more dimensions. Science, 1998, 279: $1522-1524$

15 Cairns AB, Goodwin AL. Negative linear compressibility. Phys Chem Chem Phys, 2015, 17: 20449-20465

16 Naumov P, Chizhik S, Panda MK, et al. Mechanically responsive molecular crystals. Chem Rev, 2015, 115: 12440-12490

17 Uchida E, Azumi R, Norikane Y. Light-induced crawling of crystals on a glass surface. Nat Commun, 2015, 6: 7310

18 Taniguchi $\mathrm{T}$, Sugiyama $\mathrm{H}$, Uekusa $\mathrm{H}$, et al. Walking and rolling of crystals induced thermally by phase transition. Nat Commun, 2018, 9: 538

19 Nath NK, Panda MK, Sahoo SC, et al. Thermally induced and photoinduced mechanical effects in molecular single crystals-a revival. CrystEngComm, 2014, 16: 1850-1858

20 Takanabe A, Tanaka M, Johmoto $\mathrm{K}$, et al. Optical activity and optical anisotropy in photomechanical crystals of chiral salicylidenephenylethylamines. J Am Chem Soc, 2016, 138: 15066-15077

21 Krishnan BP, Sureshan KM. A spontaneous single-crystal-tosingle-crystal polymorphic transition involving major packing changes. J Am Chem Soc, 2015, 137: 1692-1696

22 Bailey RT, Cruickshank FR, Kerkoc P, et al. Thermal properties of the novel nonlinear optical crystal $\mathrm{N}$-methylurea. J Appl Phys, 1995, 78: 3102-3106

23 Mallamace F, Branca C, Broccio M, et al. The anomalous behavior of the density of water in the range $30 \mathrm{~K}<T<373 \mathrm{~K}$. Proc Natl Acad Sci USA, 2007, 104: 18387-18391

24 Huang Y, Zhang X, Ma Z, et al. Hydrogen-bond relaxation dynamics: Resolving mysteries of water ice. Coord Chem Rev, 2015, 285: $109-165$

25 Chen Y, Kushner AM, Williams GA, et al. Multiphase design of autonomic self-healing thermoplastic elastomers. Nat Chem, 2012, 4: $467-472$

26 Ying $\mathrm{H}$, Zhang Y, Cheng J. Dynamic urea bond for the design of reversible and self-healing polymers. Nat Commun, 2014, 5: 3218

27 Steiner T. The hydrogen bond in the solid state. Angew Chem Int Ed, 2002, 41: 48-76

28 Fan CJ, Huang ZC, Li B, et al. A robust self-healing polyurethane elastomer: From H-bonds and stacking interactions to well-defined microphase morphology. Sci China Mater, 2019, 62: 1188-1198

29 Zakharov BA, Boldyreva EV. High pressure: A complementary tool for probing solid-state processes. CrystEngComm, 2019, 21: 10-22

Acknowledgements This work was supported by the National Natural Science Foundation of China (21725304, 11774120 and 11904010), Chang Jiang Scholars Program of China (T2016051), Changbai Mountain Scholars Program (2013007), the Natural Science Foundation of 
Hebei Province (A2019409043 and A2020409002), the Foundation of Hebei Educational Committee (QN2020108), and the Open Project of the State Key Laboratory of Superhard Materials, Jilin University (201915). Mr. Zhiwei Shen is acknowledged for his assistance in partial high-pressure SXRD experiments.

Author contributions Zou B conceived and supervised the project; Qiao Y prepared the DAC samples; Fan C conducted the high-pressure and low-temperature SXRD experiments and analyzed the data; Qiao Y conducted the other high-pressure experiments and analyzed the data; Qiao Y wrote the manuscript in consultation with Li Y, Wang $\mathrm{K}$ and Zou B.

Conflict of interest The authors declare that they have no conflict of interest.

Supplementary information Experimental details and supporting data are available in the online version of the paper.

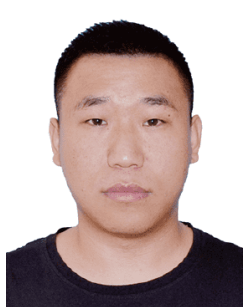

Yuancun Qiao received his $\mathrm{PhD}$ degree from the State Key Laboratory of Superhard Materials at Jilin University in 2016. He is currently a lecturer at the North China Institute of Aerospace Engineering. His research mainly focuses on highpressure investigating and controlling the properties (especially mechanical properties) of abnormal pressure-responsive materials.

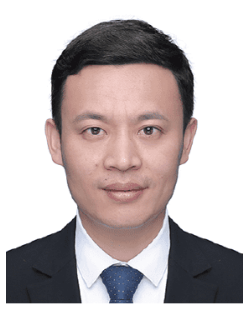

Kai Wang received his $\mathrm{PhD}$ degree in 2011, and joined the State Key Laboratory of Superhard Materials at Jilin University as a lecturer. He worked as a visiting scholar at Stanford University during 2018-2019, and was promoted to full professor in 2019. His research interests mainly focus on the properties of abnormal pressure-responsive materials. He successfully found abnormal pressure responses including giant negative linear compressibility and near zero area compressibility, pressure-induced fluorescence enhancements of a class of materials, and continuous and precise modulation of emission and absorption spectra in a large scale.

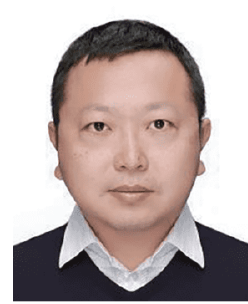

Bo Zou is a Professor of the State Key Laboratory of Superhard Materials, College of Physics, Jilin University, China. He was appointed as Chang Jiang Scholar \& National Distinguished Young Scholar. He is mainly engaged in high-pressure physics and chemistry. He resolved the ambient controversial scientific issues by means of high pressure. His scientific interests focus on the properties of abnormal pressure-responsive materials. He proposed the new concept of pressureinduced emission (PIE).

\section{分子晶体颗粒中各向同性压力驱动的集体性自愈 合反应}

乔元存 ${ }^{1,2 \dagger}$, 范长增 ${ }^{3 \dagger}$, 李欲伟 ${ }^{2}$, 王凯 ${ }^{1 *}$, 邹勃 ${ }^{1 *}$

摘要 自愈合材料具有修复损坏并改善产品寿命、安全性和功能 的能力. 但自愈合效应在有序分子晶体中却很少被报道, 且自愈效 率较低. 在本研究中, 我们首次报告了常温下 $N$-甲基艮(NMU)晶体 颗粒中各向同性压力触发的集体性自愈行为. 其中, 自愈率最高可 以达到 $100 \%$, 并伴随着“粉晶-单晶”转变. 自愈后的晶体会在更高压 力作用下破裂, 并在卸压过程中再次愈合. 原位光学显微镜、单晶 X射线衍射、粉末晶体X射线衍射和拉曼实验表明, 在 $0.3 \mathrm{GPa}$ 附近 的各向异性相变和晶粒间的紧密接触是 $N M U$ 晶体颗粒产生集体性 自愈响应的两个先决条件. 该集体性自愈行为的高自愈率来源于 在自愈合过程中外部压力被持续不断地消耗并转化为氢键重组的 化学能, 这也为提高其他分子晶体中自愈合效率提供了思路. 